\title{
Molecular simulation of hydrogen diffusion in interpenetrated metal-organic frameworks
}

\author{
Bei Liu, ${ }^{a}$ Qingyuan Yang, ${ }^{b}$ Chunyu Xue, ${ }^{b}$ Chongli Zhong* ${ }^{b}$ and Berend Smit ${ }^{c d}$ \\ Received 28th January 2008, Accepted 10th March 2008 \\ First published as an Advance Article on the web 8th April 2008 \\ DOI: $10.1039 / \mathbf{b 8 0 1 4 9 4 a}$
}

In this work a combined molecular dynamics simulation and dynamically corrected transitionstate theory (dcTST) study was performed to investigate the effect of interpenetration (catenation) on hydrogen diffusion in metal-organic frameworks (MOFs) as well as their relationships. The results on 10 isoreticular MOFs (IRMOFs) with and without interpenetration show that catenation can reduce hydrogen diffusivity by a factor of 2 to 3 at room temperature, and for the interpenetrated IRMOFs with multi-pores of different sizes, free volume can serve as a measure for hydrogen diffusivity: the bigger the free volume, the larger the hydrogen diffusivity. In addition, the present work shows that dcTST can directly reveal the influence of the MOF structure on hydrogen diffusivity, which is a powerful tool for providing a better understanding of the relationship between gas diffusivity and MOF structure.

\section{Introduction}

Metal-organic frameworks (MOFs) have been recognized as a new family of nanoporous materials that offer promising applications in gas storage, separation and catalysis etc. ${ }^{1}$ Among the various MOFs synthesized, they can be divided generally into two groups: MOFs with non-interpenetrated frameworks ${ }^{2}$ and those with interpenetrated ones. ${ }^{3}$ Apart from substantial experimental studies on the gas adsorption and diffusion in non-interpenetrated MOFs, ${ }^{4}$ there are also many theoretical works on them. ${ }^{5}$ For example, Frost et al. investigated the influencing factors on the hydrogen uptake in IRMOFs by using the grand-canonical Monte Carlo (GCMC) simulation method ${ }^{6}$ and Ramsahye et al. studied the breathing effect of the MIL-53 and MIL-47 frameworks on $\mathrm{CO}_{2}$ adsorption; ${ }^{7}$ the hydrogen adsorption sites in $\mathrm{Zn}-\mathrm{MOF}$ s were clarified by both quantum chemical ${ }^{8}$ and classical simulation studies. ${ }^{9}$ In addition, Skoulidas and Sholl investigated the self- and transport diffusion of several light gases in MOFs in detail using the molecular dynamics method, ${ }^{10}$ and framework-flexibility effects on gas adsorption and diffusion in $\mathrm{Zn}-\mathrm{MOF}$ were investigated by several groups. ${ }^{11,12}$ Nevertheless, the investigations into interpenetrated MOFs are much less. On the other hand, several available investigations have indicated that interpenetration (catenation) can enhance the adsorption capacity of MOFs, ${ }^{13}$ attributed to the additional adsorption sites and small pores formed by interpenetration that can strengthen the overall interaction between gas molecules and the pore walls. ${ }^{14}$

\footnotetext{
${ }^{a}$ Van't Hoff Institute for Molecular Sciences, University of Amsterdam, Nieuwe Achtergracht 166, 1018 WV Amsterdam, The Netherlands

${ }^{b}$ Department of Chemical Engineering, Beijing University of Chemical Technology, Beijing, 100029, China. E-mail: zhongcl@mail.buct.edu.cn

${ }^{c}$ Department of Chemical Engineering, University of California, Berkeley, CA 94720, USA

${ }^{d}$ Centre Européen de Calcul Atomique et Moléculaire (CECAM), Ecole Normale Supérieure, 46 allée d'Italie, 69007 Lyon, France
}

Despite the potential applications of interpenetrated MOFs to gas storage and separation, little is known about gasdiffusion characteristics in such MOFs. To the best of our knowledge, neither experimental nor theoretical investigations have been performed on this topic, thus this work carried out a combined molecular dynamics simulation and dynamically corrected transition-state theory (dcTST) study to investigate the effect of catenation on gas diffusion. Considering that MOFs are a family of promising materials for hydrogen storage, hydrogen was selected as the probe molecule. Therefore, this work also provides useful information for developing new MOFs as efficient hydrogen-storage materials.

\section{Models and computational method}

\subsection{MOF structures}

In this work, ten isoreticular metal-organic frameworks (IRMOFs) as synthesized by Eddaoudi et al. ${ }^{15}$ were adopted as representatives of MOFs. The guest-free framework structures of the IRMOFs were constructed from their corresponding experimental single-crystal X-ray diffraction (XRD) data ${ }^{15}$ using Materials Studio Visualizer, ${ }^{16}$ as shown in Fig. 1. As can be seen from Fig. 1, IRMOFs-1, 8, 10, 12, 14 and 16 feature the same primitive cubic topology with the octahedral $\mathrm{Zn}_{4} \mathrm{O}\left(\mathrm{CO}_{2}\right)$ clusters linked by different organic dicarboxylate linkers, while IRMOFs-9, 11, 13 and 15 are the respective interpenetration counterparts of IRMOFs-10, 12, 14 and 16. The structural properties for these IRMOF materials are given in Table 1, where the free volumes were calculated using a probe size of $0.0 \AA$ to determine the total free volume not occupied by the framework atoms.

\subsection{Force fields and the parameters}

Force field plays an important role in molecular simulations. In this work, the Coulombic interactions were not considered in our simulations as they were demonstrated to have little 

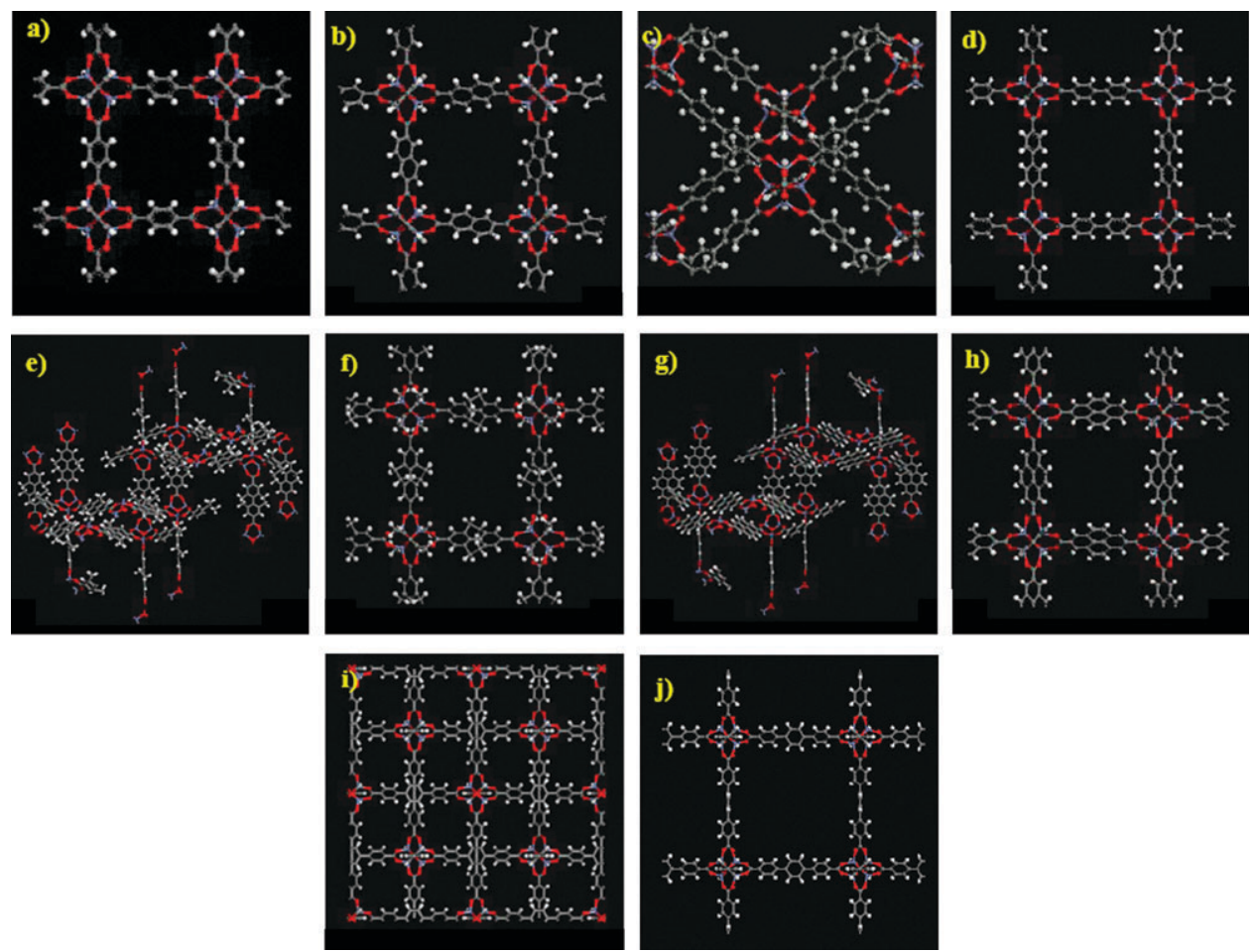

Fig. 1 Unit cell crystal structures of the IRMOFs used in the simulation: (a) IRMOF-1, (b) IRMOF-8, (c) IRMOF-9, (d) IRMOF-10, (e) IRMOF-11, (f) IRMOF-12, (g) IRMOF-13, (h) IRMOF-14, (i) IRMOF-15, (j) IRMOF-16 (Zn, blue; O, red; C, gray; H, white).

effect on the hydrogen-adsorption properties at room temperature. ${ }^{17} \mathrm{H}_{2}$ was modeled as a rigid diatomic molecule with a bond length of $0.74 \AA$. Each $\mathrm{H}$ atom in an $\mathrm{H}_{2}$ molecule was represented as a Lennard-Jones $(\mathrm{LJ})$ interaction site, for which the LJ potential parameters were taken from the force field developed in our previous work, ${ }^{18}$ and listed in Table 2. These potential parameters were optimized to reproduce the experimental PVT curve of bulk hydrogen, ${ }^{19}$ and could describe the experimental self-diffusion coefficients of bulk hydrogen well. ${ }^{18}$ In addition, this force field has also been successfully used to simulate the adsorption of $\mathrm{H}_{2}$ in MOFs by other researchers. $^{20}$

For the IRMOFs studied here, an atomistic representation was used to model all of them. The same potential model, that is the site-site LJ potential was also used to calculate the interactions between adsorbate molecules and the atoms in the frameworks of adsorbents. The potential parameters listed in
Table 2 were taken from the universal force field (UFF) of Rappé et al., ${ }^{21}$ which has been successfully employed to depict the adsorption, ${ }^{17,22}$ diffusion $^{10,23}$ and separation ${ }^{24}$ of several light gases and their mixtures in MOFs. In our simulations, all the $\mathbf{L} \mathbf{J}$ cross-interaction parameters were determined by the Lorentz-Berthelot mixing rules.

\subsection{Molecular dynamics simulation}

In this work, equilibrium molecular dynamics (MD) simulations were carried out in the canonical $(N V T)$ ensemble to investigate the effects of pore size and catenation on the diffusion behaviors of $\mathrm{H}_{2}$ in MOFs. The whole MD simulations were performed under room temperature, and the NoséHoover chain (NHC) thermostat as formulated by Martyna et l. $^{25}$ was used to maintain the constant-temperature condition. Similar to previous works, ${ }^{5,6,9-12}$ all the IRMOFs studied in this work were treated as rigid with atoms frozen at their

Table 1 Structural properties for the IRMOFs studied in this work

\begin{tabular}{|c|c|c|c|c|c|c|c|}
\hline Material & Pore shape $^{a}$ & Unit cell/Å & Cell angle ${ }^{\circ}$ & $d_{\text {pore }}{ }^{a} / \AA$ & $\begin{array}{l}\rho_{\text {crys }}{ }^{a} / \\
\mathrm{g} \mathrm{cm}^{-3}\end{array}$ & $\begin{array}{c}V_{\text {free }}{ }^{b} / \\
\mathrm{cm}^{3} \mathrm{~g}^{-1}\end{array}$ & $\begin{array}{l}\text { Porosity } \\
(\%)\end{array}$ \\
\hline IRMOF-1 & Cubic & $a=b=c=25.832$ & $\alpha=\beta=\gamma=90$ & $10.9 / 14.3$ & 0.59 & 1.36 & 80.5 \\
\hline IRMOF-8 & Cubic & $a=b=c=30.092$ & $\alpha=\beta=\gamma=90$ & $12.5 / 17.1$ & 0.45 & 1.87 & 84.0 \\
\hline IRMOF-10 & Cubic & $a=b=c=34.281$ & $\alpha=\beta=\gamma=90$ & $16.7 / 20.2$ & 0.33 & 2.66 & 87.9 \\
\hline IRMOF-12 & Cubic & $a=b=c=34.281$ & $\alpha=\beta=\gamma=90$ & $13.9 / 20.0$ & 0.38 & 2.24 & 85.1 \\
\hline IRMOF-14 & Cubic & $a=b=c=34.381$ & $\alpha=\beta=\gamma=90$ & $14.7 / 20.1$ & 0.37 & 2.30 & 86.3 \\
\hline IRMOF-16 & Cubic & $a=b=c=42.980^{c}$ & $\alpha=\beta=\gamma=90$ & 23.3 & 0.21 & 4.46 & 91.5 \\
\hline IRMOF-9 & Cubic/catenation & $a=17.147, b=23.322, c=25.255$ & $\alpha=\beta=\gamma=90$ & $4.5 / 6.3 / 8.1 / 10.7$ & 0.66 & 1.14 & 74.8 \\
\hline IRMOF-11 & Cubic/catenation & $a=b=24.822, c=56.734$ & $\alpha=\beta=90, \gamma=120$ & $3.5 / 3.8 / 4.7 / 6.1 / 7.3 / 11.1$ & 0.76 & 0.92 & 69.8 \\
\hline IRMOF-13 & Cubic/catenation & $a=b=24.822, c=56.734$ & $\alpha=\beta=90, \gamma=120$ & $4.2 / 4.7 / 6.1 / 7.0 / 11.4$ & 0.75 & 0.95 & 71.4 \\
\hline IRMOF-15 & Cubic/catenation & $a=b=c=42.918^{c}$ & $\alpha=\beta=\gamma=90$ & - & 0.41 & 2.01 & 82.8 \\
\hline
\end{tabular}


Table 2 LJ potential parameters for $\mathrm{H}_{2}$ and the IRMOFs used in this work

\begin{tabular}{lrrrrr}
\hline LJ parameters & $\mathrm{H}_{2} \_\mathrm{H}$ & MOF_O & MOF_C & MOF_H & MOF_Zn \\
\hline$\sigma / \AA$ & 2.72 & 3.12 & 3.43 & 2.57 & 2.46 \\
$\varepsilon / k_{\mathrm{B}} / \mathrm{K}$ & 10.00 & 30.19 & 52.84 & 22.14 & 62.40 \\
\hline
\end{tabular}

crystallographic positions during simulations. Although the diffusion properties of guest molecules may depend significantly on the lattice dynamics of MOFs, ${ }^{12}$ the interactions between $\mathrm{H}_{2}$ molecules and the frameworks of MOFs are weak and our purpose is to perform a comparative study on IRMOFs with and without catenation; thus the treatment of rigid MOFs is reasonable. The simulation cell consists of $2 \times 2$ $\times 2$ to $4 \times 4 \times 4$ elementary cells of the IRMOF crystal lattice to ensure at least fifty $\mathrm{H}_{2}$ molecules are accommodated in the cell, and the structural model for a unit cell of each IRMOF was constructed using the experimental XRD data as shown in Fig. 1. The velocity Verlet algorithm was used to integrate Newton's equations of motion. The time step used in the MD simulations was taken as $1.0 \mathrm{fs}$. All the $\mathrm{LJ}$ interactions were calculated using the cut-and-shifted potential with a $17.0 \AA$ cutoff radius, and periodic boundary conditions were applied in all three dimensions. Simulations were performed as follows: molecules were randomly inserted into the IRMOF lattices, and then relaxed using approximately $100000 \mathrm{NVT}$ Monte Carlo moves. Following the relaxation, velocities from the Maxwell-Boltzmann distribution at the required temperature were assigned to all the adsorbate molecules, and the total momentum of the system was set to zero. Next, the simulation systems were allowed to equilibrate with $100000 \mathrm{MD}$ steps before the final simulations were run for $2000000 \mathrm{MD}$ steps to sample the diffusion properties of interest. At least 10 independent simulations were performed for each loading to estimate the statistical error. During each simulation, the trajectory of the system was saved every 100 steps to subsequently calculate the self-diffusion coefficient $D_{\mathrm{s}}$ by a meansquare displacements (MSD) method using a so-called order- $N$ algorithm. ${ }^{26}$ It was checked that MD simulations conducted in the microcanonical $(N V E)$ ensemble gave equivalent results. To increase the computational efficiency, the potential energies and forces between the adsorbate and adsorbent were initially tabulated on a series of three-dimensional grid points with grid spacing $0.15 \AA$. During the simulations, the potential energy and force at any position in the adsorbent were determined by interpolation. ${ }^{23}$

\subsection{Dynamically corrected transition-state theory}

Dynamically corrected transition-state theory $(\mathrm{dcTST})^{27}$ regards diffusion processes in confinement as hopping events on a lattice, where the hopping from state $\mathrm{A}$ to another state B is limited by a free energy barrier between the two states. A and $\mathrm{B}$ are separated by a lattice distance $\lambda$. Together with the dynamical correction factor $\kappa$, free energy profiles $F(q)$ can be used to compute a hopping rate $k_{\mathrm{A} \rightarrow \mathrm{B}}$ between states $\mathrm{A}$ and $\mathrm{B}$ by:

$$
k_{\mathrm{A} \rightarrow \mathrm{B}}=\kappa \times \sqrt{\frac{k_{\mathrm{B}} T}{2 \pi m}} \times \frac{\exp [-\beta F(q *)]}{\int_{\text {pore }} \exp [-\beta F(q)] \mathrm{d} q}
$$

where $k_{\mathrm{B}}$ is Boltzmann's constant, $T$ is the temperature of the system, $m$ is the mass of the hopping particle, and $q^{*}$ denotes the (assumed) location of the barrier. This hopping rate $k_{\mathrm{A} \rightarrow \mathrm{B}}$ can in turn be converted to a self-diffusion coefficient $D_{\mathrm{s}}$ via:

$$
\begin{aligned}
D_{\mathrm{S}} & =k_{\mathrm{A} \rightarrow \mathrm{B}} \times \lambda^{2} \\
& =\kappa \times \sqrt{\frac{k_{\mathrm{B}} T}{2 \pi m}} \times \frac{\exp [-\beta F(q *)]}{\int_{\text {pore }} \exp [-\beta F(q)] \mathrm{d} q} \times \lambda^{2} \\
& =\kappa \times D_{\mathrm{s}}^{\mathrm{TST}}
\end{aligned}
$$

In the above equation, $D_{\mathrm{s}}^{\mathrm{TST}}$ is the free-energy contribution to the self-diffusion coefficient, the part of the diffusion that is governed by free energy barriers: influences of the confinement topology on the diffusion of the gas molecules. $\kappa$ is regarded as a measure of the interaction between the fluid particles themselves. ${ }^{28}$

To compute free-energy profiles, we performed $N V T$-ensemble Monte Carlo simulations using the histogram-sampling (HS) method. ${ }^{27}$ In the HS method, a histogram is made of the particle positions, mapped on the reaction coordinate $q$. Then this histogram can be converted into a free-energy profile by using $\beta F(q)=-\ln \langle P(q)\rangle$, where $P(q)$ denotes the probability of finding a molecule at a given position $q$ according to the histogram. Details of the calculations can be found elsewhere. ${ }^{29}$

\section{Results and discussion}

\subsection{Effect of catenation on hydrogen diffusivity in MOFs}

To understand how the pore size and interpenetration (catenation) affect the hydrogen-diffusion behavior, the self-diffusivities $\left(D_{\mathrm{s}}\right)$ of hydrogen in the ten selected IRMOFs at room temperature were examined with a MD simulation. The simulation results are shown in Fig. 2, as a function of hydrogen loading. Fig. 2a shows that for the six non-interpenetrated IRMOF materials with the same primitive cubic topology and loading, the self-diffusivities of hydrogen have the following order: IRMOF-16 > IRMOF-10 > IRMOF-14 (or IRMOF-12) > IRMOF-8 > IRMOF-1, which is consistent with the gradually decreasing sequence of pore size of the IRMOF materials. In Fig. 2a it seems the dependency of hydrogen diffusivity in IRMOF-16 is more evident than that in the other five IRMOFs; this can be explained as follows: (1) the porosity of IRMOF-16 (Table 1) is larger than the other five IRMOFs, thus at the same loading (mg per total volume of material) the hydrogen density in IRMOF-16 is smaller than that in the other materials, and (2) moreover, the pore size of IRMOF-16 is much larger than that of the other five IRMOFs, leading to a much larger hydrogen diffusivity at the same loading. The interplay of the above two factors results in a very evident loading dependency of hydrogen diffusivity as shown in Fig. 2 within the loadings studied.

Fig. $2 b$ shows the effect of catenation on the self-diffusivities of hydrogen in the IRMOFs at room temperature. The hydrogen self-diffusivities in the IRMOFs (IRMOF-10, IRMOF-12, IRMOF-14 and IRMOF-16) without catenation are two to three times of those in their corresponding catenated 

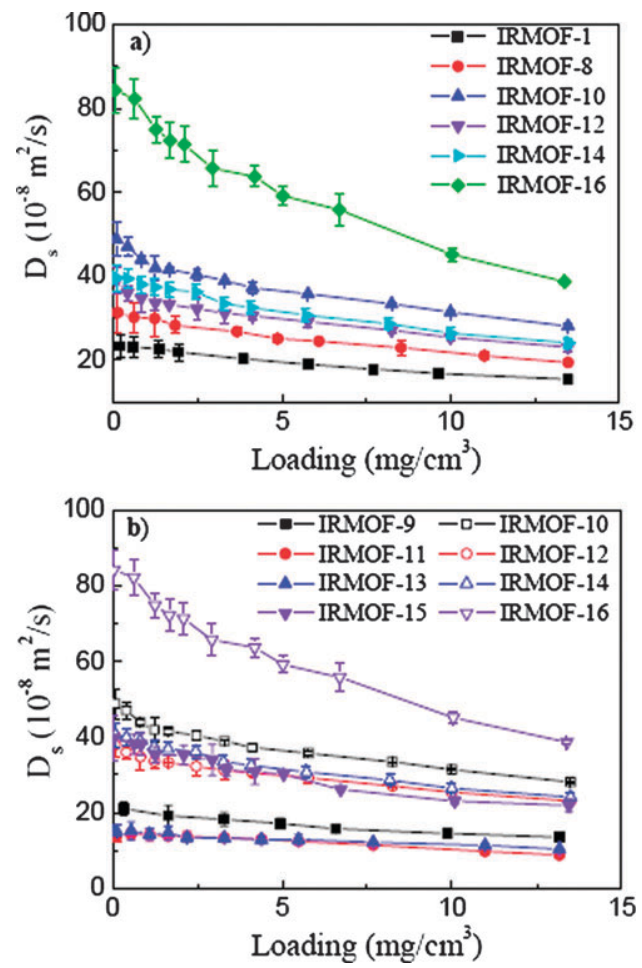

Fig. 2 (a) Comparison of the self-diffusivities of hydrogen in IRMOFs with the same primitive cubic topology at room temperature. (b) Effect of catenation on the self-diffusivities of hydrogen in the IRMOFs at room temperature.

counterparts (IRMOF-9, IRMOF-11, IRMOF-13 and IRMOF-15 respectively). This implies that the motion of hydrogen molecules in MOFs is largely restricted by their catenation structures. In the interpenetrated IRMOFs, there are various pores with different sizes ( 4 in IRMOF-9 and 6 in IRMOF-11) and thus it is difficult, if not impossible, to correlate hydrogen diffusivity to pore size. However, an examination of the free volume of each material and the hydrogen diffusivity values shows that there is a good correlation between them: the larger the free volume, the bigger the hydrogen diffusivity. To illustrate this more intuitively, the self-diffusivities of hydrogen at infinite dilution loading were plotted against the free volumes of the interpenetrated IRMOF materials as well as those without catenation. The results presented in Fig. 3 show that a good correlation does exist between them. Similar relationships also hold at other hydrogen loadings. This leads us to the conclusion that for the interpenetrated IRMOFs with multi-pores of different sizes, material free volume can serve as a measure of hydrogen diffusivity.

To understand the occupying situation of hydrogen molecules in the studied MOFs, center-of-mass (COM) probability distributions of hydrogen in IRMOF-10 and its catenation counterpart IRMOF-9 at the loading of 10 molecules per unit cell and room temperature were calculated, and the results are shown in Fig. 4. Obviously, in IRMOF-10 hydrogen molecules distribute nearly evenly in the metal cluster and linker region, with slight accumulation near the cluster area, while in IRMOF-9 the situation is a little different with the largest accumulation occurring in the catenated area formed by the

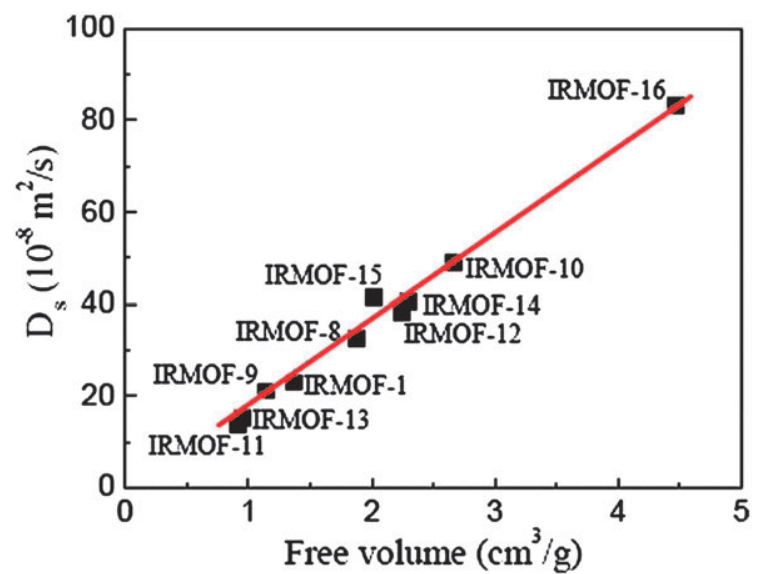

Fig. 3 Self-diffusivities of hydrogen at infinite dilution loading vs. free volume of the IRMOFs.

metal clusters. This confirms that catenation can improve gas affinity at room temperature.

\subsection{The relationship between hydrogen diffusivity and MOF structure by dcTST}

To further understand the relationship between hydrogen diffusivity and MOF structure, a dynamically corrected transition-state theory (dcTST) ${ }^{27-29}$ study was performed for IRMOF-16 and its catenation counterpart IRMOF-15 at room temperature and infinite dilution. The diffusion at infinite dilution in general can be fully accounted for by $D_{\mathrm{s}}^{\mathrm{TST}}$, i.e. the free-energy contribution to the self-diffusion, as the dynamical correction factor $\kappa$ is the contribution to the diffusion of interparticle collisions. ${ }^{28}$ The free-energy profiles in IRMOF15 and 16 are shown in Fig. 5. The reaction coordinate $q$ is simply the position of a tagged molecule along the Cartesian connection from one pore to another. Because the structures of IRMOF-15 and 16 are highly symmetric, we only showed the free-energy profiles along the $z$-direction as an example. The wells of the barriers correspond to the locations near the metal cluster and linker region inside the pores, i.e. the energetically favorable adsorption sites, and the maxima correspond to the locations of the metal cluster and linker region. Obviously, the number of barriers per unit cell in IRMOF-15 is larger than that in IRMOF-16, which is attributed to the catenation structure in the former. From our MD simulations we know that hydrogen diffusivity in IRMOF-15 is about half of that in IRMOF-16 (39.8 and 84.3 respectively) at infinite dilution, this can be explained by comparing the $D_{\mathrm{s}}^{\mathrm{TST}}$ for the two materials from dcTST. According to eqn (2), the $D_{\mathrm{s}}^{\mathrm{TST}}$ is given as $D_{\mathrm{s}}^{\mathrm{TST}}=\sqrt{\frac{k_{\mathrm{B}} T}{2 \pi m}} \times \frac{\exp [-\beta F(q *)]}{\int_{\text {pore }} \exp [-\beta F(q)] \mathrm{d} q} \times \lambda^{2}$. Obviously, the values of $\sqrt{\frac{k_{\mathrm{B}} T}{2 \pi m}} \times \exp [-\beta F(q *)]$ are nearly identical for IRMOF-15 and IRMOF-16 since the barrier heights are nearly same for the two materials. On the other hand, $\left[\int_{\text {pore }} \exp [-\beta F(q)] \mathrm{d} q\right]_{\mathrm{IRMOF}-15} \approx 0.5\left[\int_{\text {pore }} \exp [-\beta F(q)] \mathrm{d} q\right]_{\text {IRMOF-16 }}$ and $\lambda_{\text {IRMOF-15 }}$ (the lattice distance between two adjacent pore centers) $=0.5 \lambda_{\text {IRMOF-16}}$. Therefore, the dcTST gives results consistent with the MD simulations, while the structural effects 


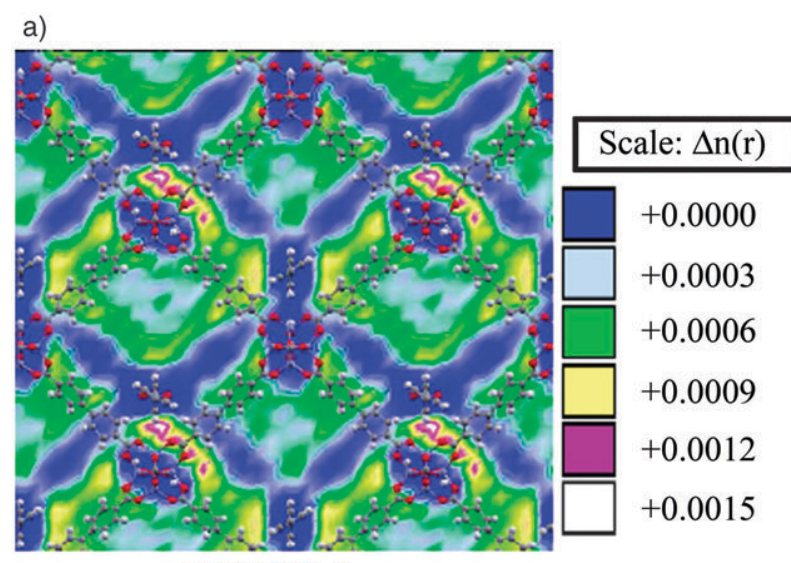

IRMOF-9

b)

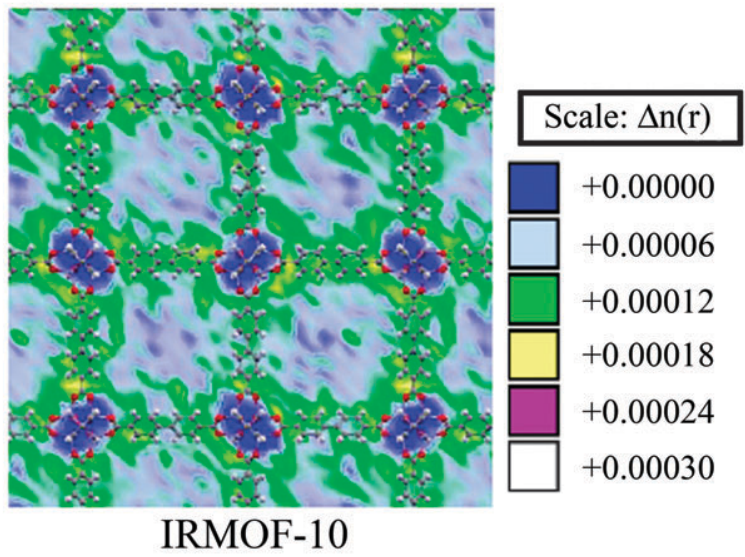

Fig. 4 Contour plots of COM probability density in planes (a) through the catenated area in IRMOF-9 and (b) through the $\mathrm{Zn}_{4} \mathrm{O}$ clusters in IRMOF-10 (Zn, blue; O, red; C, gray; H, white).

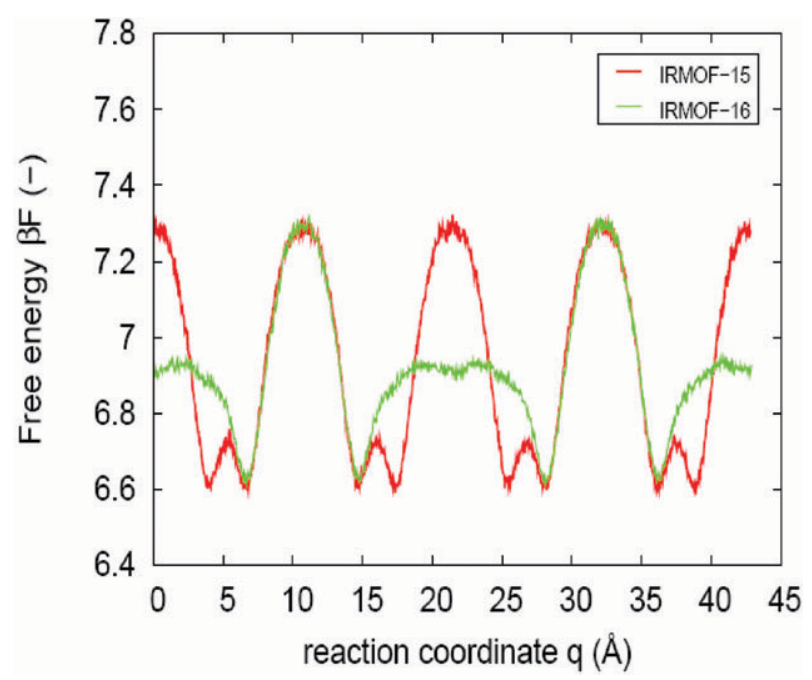

Fig. 5 Free-energy profiles $F(q)$ of hydrogen in IRMOF-15 and IRMOF-16 at room temperature and infinite dilution. The reaction coordinate is chosen along the $z$-direction. For IRMOF-15 the pore centers are located at 5.36, 16.09, 26.82 and $42.92 \AA$, while for IRMOF-16 the pore centers are located at $0,21.49$ and $42.98 \AA$. can be revealed more clearly by the former: catenation in IRMOF-15 leads to a decrease of $\lambda$, i.e. at a given length the number of barriers is increased, resulting in a smaller hydrogen diffusivity in IRMOF-15 than in IRMOF-16. The above analysis shows that dcTST is a useful tool for understanding the relationship between hydrogen diffusivity and MOF structure and it may find wide applications in future studies of MOFs.

\section{Conclusion}

This work found that free volume plays the main role in determining hydrogen diffusivity in MOFs at room temperature, and that for interpenetrated MOFs with multi-pores of different sizes, free volume can serve as a measure for hydrogen diffusivity. The results on the ten IRMOFs show that catenation can reduce hydrogen diffusivity by a factor of 2 to 3 at room temperature, attributed mainly to the increase of the number of barriers at a given space. In addition, the present work shows that dcTST can characterize the number and value of diffusion barriers in MOFs to relate hydrogen diffusivity directly to the structure of a MOF material, giving a better understanding of guest-molecule diffusivity in MOFs.

\section{Acknowledgements}

The financial supports of the NSFC (No. 20725622 and 20706002), the Dutch STW/CW Separation Technology program (700.56.655-DPC.6243), and the EC through the Marie Curie EXT project MEXT-CT-2005-023311 are greatly appreciated.

\section{References}

1 J. L. C. Rowsell and O. M. Yaghi, Angew. Chem., Int. Ed., 2005, 44, 4670-4679; R. Q. Snurr, J. T. Hupp and S. T. Nguyen, AIChE J., 2004, 50, 1090-1095; U. Mueller, M. Schubert, F. Teich, H. Puetter, K. Schierle-Arndt and J. Pastre, J. Mater. Chem., 2006, 16, 626-636.

2 Y. Kubota, M. Takata, R. Matsuda, R. Kitaura, S. Kitagawa, K. Kato, M. Sakata and T. C. Kobayashi, Angew. Chem., Int. Ed., 2005, 44, 920-923; S. S. Kayes and J. R. Long, J. Am. Chem. Soc., 2008, 130, 806-807.

3 B. Kesanli, Y. Cui, M. R. Smith, E. W. Bittner, B. C. Bockrath and W. Lin, Angew. Chem., Int. Ed., 2005, 44, 72-75; B. Chen, S. Ma, E. J. Hurtado, E. B. Lobkovsky and H. C. Zhou, Inorg. Chem., 2007, 46, 8490-8492.

4 M. Latroche, S. Surblé, C. Serre, C. Mellot-Draznieks, P. L. Llewellyn, J. H. Lee, J. S. Chang, S. H. Jhung and G. Férey, Angew. Chem., Int. Ed., 2006, 45, 8227-8231; A. G. Wong-Foy, A. J. Matzger and O. M. Yaghi, J. Am. Chem. Soc., 2006, 128, 3494-3495; S. S. Kaye, A. Dailly, O. M. Yaghi and J. R. Long, J. Am. Chem. Soc., 2007, 129, 14176-14177.

5 J. L. Belof, A. C. Stern, M. Eddaoudi and B. Space, J. Am. Chem. Soc., 2007, 129, 15202-15210; K. S. Walton, A. R. Millward, D. Dubbeldam, H. Frost, J. J. Low, O. M. Yaghi and R. Q. Snurr, J. Am. Chem. Soc., 2008, 130, 406-407; Q. Yang, C. Xue, C. Zhong and J. Chen, AIChE J., 2007, 53, 2832-2840.

6 H. Frost, T. Düren and R. Q. Snurr, J. Phys. Chem. B, 2006, 110, 9565-9570.

7 N. A. Ramsahye, G. Maurin, S. Bourrelly, P. Llewellyn, T. Loiseau and G. Férey, Phys. Chem. Chem. Phys., 2007, 9, 1059-1063; N. A. Ramsahye, G. Maurin, S. Bourrelly, P. L. Llewellyn, T. Loiseau, C. Serrec and G. Férey, Chem. Commun., 2007, 3261-3263.

8 E. Klontzas, A. Mavrandonakis, G. E. Froudakis, Y. Carissan and W. Klopper, J. Phys. Chem. C, 2007, 111, 13635-13640; Q. Yang 
and C. Zhong, J. Phys. Chem. B, 2006, 110, 655-658; T. B. Lee, D. Kim, D. H. Jung, S. B. Choi, J. H. Yoon, J. Kim, K. Choi and S. H. Choi, Catal. Today, 2007, 120, 330-335.

9 S. S. Han, W. Q. Deng and W. A. Goddard III, Angew. Chem., Int. Ed., 2007, 46, 6289-6292; D. Dubbeldam, H. Frost, K. S. Walton and R. Q. Snurr, Fluid Phase Equilib., 2007, 261, 152-161.

10 A. I. Skoulidas and D. S. Sholl, J. Phys. Chem. B, 2005, 109, $15760-15768$.

11 J. A. Greathouse and M. D. Allendorf, J. Am. Chem. Soc., 2006, 128, 10678-10679; D. Dubbeldam, K. S. Walton, D. E. Ellis and R. Q. Snurr, Angew. Chem., Int. Ed., 2007, 46, 4496-4499.

12 S. Amirjalayer, M. Tafipolsky and R. Schmid, Angew. Chem., Int. Ed., 2007, 46, 463-466.

13 J. L. C. Rowsell and O. M. Yaghi, J. Am. Chem. Soc., 2006, 128, 1304-1315; D. H. Jung, D. Kim, T. B. Lee, S. B. Choi, J. H. Yoon, J. Kim, K. Choi and S. H. Choi, J. Phys. Chem. B, 2006, 110, 22987-22990; B. Chen, S. Ma, F. Zapata, E. B. Lobkovsky and J. Yang, Inorg. Chem., 2006, 45, 5718-5720.

14 S. Ma, D. Sun, M. Ambrogio, J. A. Fillinger, S. Parkin and H. C. Zhou, J. Am. Chem. Soc., 2007, 129, 1858-1859.

15 M. Eddaoudi, J. Kim, N. Rosi, D. Vodak, J. Wachter, M. O'Keeffe and O. M. Yaghi, Science, 2002, 295, 469-472.

16 Materials Studio 3.0 V, Accelrys Inc., San Diego, CA, 2003.

17 G. Garberoglio, A. I. Skoulidas and J. K. Johnson, J. Phys. Chem. $B, 2005,109,13094-13103$.

18 Q. Yang and C. Zhong, J. Phys. Chem. B, 2005, 109, 11862-11864.
19 N. B. Vargaftik, Tables of Thermophysical Properties of Liquids and Gases, John Wiley \& Sons, New York, 1975.

20 S. Surblé, F. Millange, C. Serre, T. Düren, M. Latroche, S. Bourrelly, P. L. Llewellyn and G. Férey, J. Am. Chem. Soc., 2006, 128, 14889-14896.

21 A. K. Rappé, C. J. Casewit, K. S. Colwell, W. A. Goddard III and W. M. Skiff, J. Am. Chem. Soc., 1992, 114, 10024-10035.

22 V. Krungleviciute, K. Lask, L. Heroux, A. D. Migone, J.-Y. Lee, J. Li and A. Skoulidas, Langmuir, 2007, 23, 3106-3109.

23 A. I. Skoulidas, J. Am. Chem. Soc., 2004, 126, 1356-1357.

24 S. Keskin and D. S. Sholl, J. Phys. Chem. C, 2007, 111, 14055-14059; R. Babarao, Z. Hu, J. Jiang, S. Chempath and S. I. Sandler, Langmuir, 2007, 23, 659-666.

25 G. Martyna, M. E. Tuckerman, D. J. Tobias and M. L. Klein, Mol. Phys., 1996, 87, 1117-1157.

26 D. Frenkel and B. Smit, Understanding Molecular Simulation: From Algorithms to Applications, Academic Press, San Diego, 2002.

27 E. Beerdsen, B. Smit and D. Dubbeldam, Phys. Rev. Lett., 2004, 93, 248301; E. Beerdsen, D. Dubbeldam and B. Smit, Phys. Rev. Lett., 2005, 95, 164505; E. Beerdsen, D. Dubbeldam and B. Smit, Phys. Rev. Lett., 2006, 96, 044501.

28 E. Beerdsen, D. Dubbeldam and B. Smit, J. Phys. Chem. B, 2006, 110, 22754-22772.

29 D. Dubbeldam, E. Beerdsen, T. J. H. Vlugt and B. Smit, J. Chem. Phys., 2005, 122, 224712. 